\title{
Intestinal Protozoan Parasitosis in 4- and 11- year-old Children in Cunduana Community, Riobamba Canton, Chimborazo, June 2019 June
}

XVIII International Seminar on Health, Food and Human Nutrition

Corresponding Author:

Silvia Marcela Inca Martínez

s_inca@espoch.edu.ec

Published: 9 September 202

Production and Hosting by

Knowledge $E$

(c) Silvia Marcela Inca

Martínez et al. This article is distributed under the terms of the Creative Commons Attribution License, which permits unrestricted use and redistribution provided that the original author and source are credited.

\section{Parasitosis Intestinal por Protozoos en Niños y Niñas de 4-11 Años, Comunidad Cunduana, Cantón Riobamba, Chimborazo, Junio 2019}

Silvia Marcela Inca Martínez ${ }^{1,2}$, Martha Cecilia Bonilla Caicedo ${ }^{1}$, and Silvana Paola Ocaña Coello ${ }^{3}$

${ }^{1}$ Carrera de Medicina, Facultad de Salud Pública, Escuela Superior Politécnica de Chimborazo, Riobamba, Ecuador

${ }^{2}$ Centro de Transfusión, Hospital Provincial General Docente de Riobamba, Chile y Av. Juan Félix Proaño, Riobamba, Ecuador

${ }^{3}$ Ingeniería Ambiental, Facultad de Ciencias, Escuela Superior Politécnica de Chimborazo, Riobamba, Ecuador

\section{Abstract}

Introduction: Parasitosis is a public health problem that affects millions of people, schoolage children are more vulnerable, affects their physical and intellectual development, are transmitted by fecal-oral route, and is associated with poor hygienic and sanitary conditions. In Ecuador they constitute the second reason for medical consultation. Objective: To contribute to the epidemiological knowledge of intestinal parasitosis by protozoa in our environment. Methodology: An experimental descriptive study, with a universe of 80 children, obtaining 55 samples, a direct examination was carried out in preparations with physiological serum and lugol, identifying the parasites by their microscopic morphology. Results: The prevalence of intestinal parasitosis by protozoa was $78.18 \%$, while for the parasites Endolimax nana 30.9\% Entamoeba histolytica, 34.5\% and Giardia lamblia 14.5\%. 40\% of the participants were monoparasitized and $38.2 \%$ had polyparasitosis. Conclusion: High prevalence of parasitosis by intestinal protozoa was determined in the children of the Unit with $78.18 \%$. The most prevalent ameboid protozoan was $E$. nana, followed by the pathogenic protozoan $E$. histolytica, while G. lamblia was flagellated protozoan identified. The lack of adequate sanitary infrastructure, habits of hygiene, environmental pollution, influence the prevalence of intestinal parasitosis.

Keywords: protozoan infections, intestinal diseases, child.

\section{Resumen}

Introducción: Parasitosis es un problema de salud pública que afecta a millones de personas, los niños en edad escolar son más vulnerables, afecta su desarrollo físico e intelectual, se transmiten por vía fecal-oral, y está asociada a deficientes condiciones higiénicas y sanitarias. En Ecuador constituyen el segundo motivo de consulta médica. Objetivo: contribuir al conocimiento epidemiológico de las parasitosis intestinales por protozoos en nuestro medio. Metodología: estudio descriptivo experimental, con un universo de 80 niños y niñas, 
obteniendo 55 muestras, se realizó examen directo en preparaciones con suero fisiológico y lugol, identificando los parásitos por su morfología microscópica. Resultados: La prevalencia de parasitosis intestinal por protozoos fue de 78,18\%, mientras que para los parásitos Endolimax nana 30,9\%, Entamoeba histolytica 34,5\% y Giardia lamblia 14,5\%. El 40\% de los participantes fue monoparasitado y el 38,2\% presentó poliparasitosis. Conclusiones: Se determinó alta prevalencia de parasitosis por protozoos intestinales en los niños y niñas de la Unidad con un $78,18 \%$. El protozoo ameboideo de mayor prevalencia fue $E$. nana, seguido del protozoo patógeno $E$. histolytica, mientras que G. lambia fue protozoo flagelado identificado. La falta de una adecuada infraestructura sanitaria, hábitos de higiene, contaminación ambiental, influyen en la prevalencia de la parasitosis intestinal.

Palabras Clave: infecciones por protozoos, parasitosis intestinales, niños.

\section{Introducción}

La parasitosis es un problema de salud pública global ya que afecta a millones de persona en todo el mundo, representando el $25 \%$ de todas las enfermedades infecciosas que afecta a los humanos, se presenta en gran variedad de regiones y climas, y su diseminación no está limitada a factores económicos o geográficos lo que la ha convertido en un problema significativo a todo nivel social, presentándose en individuos de todas edades y sexo [1-3]. Las enfermedades parasitarias intestinales son más comunes en niños de edad escolar, debido a su menor nivel inmunológico y por lo tanto poseen menor tolerancia a los mismos, a lo que se suma que durante la infancia existe mayor oportunidad de mantener contacto con las formas infectantes de los parásitos, con consecuencias desfavorables en el desarrollo físico y cognitivo de los niños infectados [4-7].

La mayor parte de parásitos son transmitidos por vía fecal-oral, mediante la ingestión de agua o alimentos contaminados, aportando a la transmisión malas prácticas higiénicas, bajas condiciones socioeconómicas, falta de medidas sanitarias, comunicaciones aéreas y marítimas $[5,8,9]$.

Aproximadamente 1110 millones de personas están infectadas por cestodos, 240 millones por trematodos y 3200 millones por trematodos. Sin embargo los protozoos afectan del $20-50 \%$ de la población mundial, siendo el grupo parasitario con mayor prevalencia [8], la mortalidad por parásitos es baja, pese a ello se reportan 100.000 fallecimientos por amebiasis cada año [10]. En nuestro país las parasitosis intestinales están en segundo lugar de las principales causas de morbilidad ambulatoria que reporta el Ministerio de Salud Pública del Ecuador (MSPE), y dentro de los 10 motivos de consulta pediátrica. Según autores ecuatorianos la población infantil muestra una frecuencia de $85,7 \%$ de parasitosis intestinales especialmente en preescolares y escolares, considerándose que las poblaciones alejadas de los ciudades y grupos étnicos vulnerables, son los más afectados por parasitosis, por lo cual el MSPE en el año 2015 creó el Programa Nacional de Manejo Multidisciplinario de las Parasitosis Desatendidas 
en el Ecuador (Propad), sin que hasta el momento se hayan alcanzado los objetivos propuestos $[3,6]$.

Las parasitosis y especialmente las poliparasitosis afectan la salud de los individuos, principalmente en los niños que son el grupo más vulnerable y quienes presentan una mayor exposición a la infección parasitaria y a la reinfección, provocando cuadros digestivos que pueden ser graves con tasas altas de mortalidad [11], además un efecto negativo en su crecimiento y aprendizaje. Las infecciones parasitarias se asocian a deficientes condiciones higiénicas y sanitarias del ambiente y del lugar donde desarrollan sus actividades [12, 13].

Los protozoos considerados como patógenos Entamoeba histolytica y Giardia lamblia tienen la capacidad de trasmitirse por cuerpos de agua naturales y artificiales, utilizadas para consumo o recreación, ya sea por un inadecuado tratamiento o por contaminación con desechos fecales humanos y de animales, que contienen quistes que son la forma infectante de los parásitos [14].

La finalidad del presente trabajo es contribuir al conocimiento epidemiológico de las parasitosis intestinales por protozoos en nuestro medio, ayudando a identificar la prevalencia de estas parasitosis en muestras de heces en niños y niñas de edad escolar en la comunidad Cunduana, parroquia Licán, perteneciente al cantón Riobamba provincia de Chimborazo.

\section{Métodos}

Se trata de un estudio descriptivo experimental, aplicado en la Unidad Educativa Vicente Rocafuerte de la comunidad de Cunduana, parroquia Licán, del Cantón Riobamba, en junio de 2019. Cunduana pertenece a la región Sierra, ubicada a $2764 \mathrm{~m}$ sobre el nivel del mar, con un clima frío que varía de 8 a $19^{\circ} \mathrm{C}$ en el transcurso del año. Se trabaja con la totalidad del universo, 80 niños y niñas, de la Unidad Educativa, cuyos padres o representantes dieron el consentimiento para participar en la investigación, obteniendo muestras de niños o niñas desde Inicial II hasta séptimo año de educación básica.

A cada niño o niña se entregó una caja para recolección de muestra de heces, sin embargo, solo 55 niños y niñas entregan la muestra, durante la etapa de recolección, por lo que se toma de referencia a los 55 niños y niñas para el presente estudio, no se aplicó alguna técnica de selección muestral.

Para el análisis coproparasitario se tomaron en cuenta las muestras que estaban correctamente identificadas, con una cantidad suficiente de muestra para el análisis; excluyéndose cajas de recolección que no contenían muestra y de aquellos que no entregaron la muestra en los tiempos establecidos para la recolección.

Siguiendo el protocolo se realizó el análisis de las muestras de heces, utilizando suero fisiológico y lugol, para la preparación en fresco de cada placa, posteriormente se analizó en el microscopio con lentes de 10 y 40 x, cada preparación en campos sobre montados, observando e identificando los parásitos protozoos en base a su morfología 
típica. Los resultados observados se registraron en un formato realizado en hoja de cálculo Excel, para su análisis e interpretación en función de la revisión bibliográfica.

\section{Resultados}

De los niños de Inicial II hasta séptimo año de educación básica, se recolectaron 56 cajas para heces, de las cuales 1 no contenía muestra. Se trabajó con 55 muestras siendo 25 correspondientes al género masculino (45,5\%) y 30 al género femenino (54,5\%), como se muestra en la Figura 1 género de la población en estudio, comprendidos entre 4 y 11 años de edad.

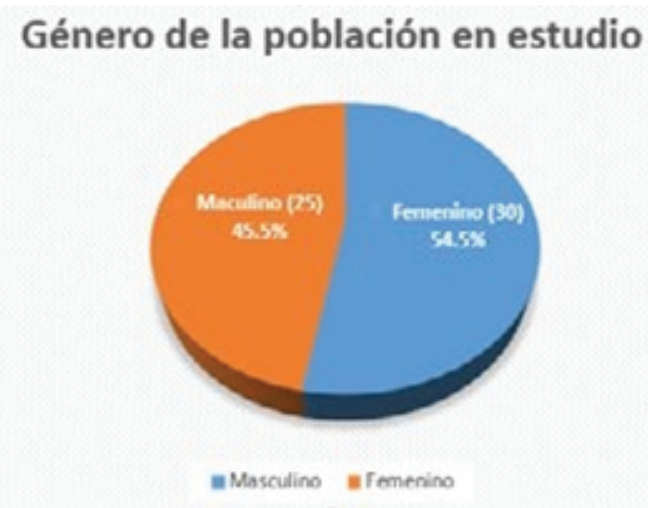

Figure 1

Género de la población en estudio. Fuente: Propia.

Las muestras analizadas por observación directa se encontraron los siguientes resultados, en 12 muestras no se observó ningún parásito protozoo. Se identificaron los siguientes protozoos ameboideos: En 25 (45,5\%) muestras se identificó quistes de Endolimax nana, mientras que 17 (30,9\%) muestras se encontró quistes de E. histolytica, mientras que en 19 (34,5\%) Quistes de E. coli. Los protozoos flagelados identificados fueron: Quistes de G. lamblia en 8 (14,5\%) muestras y en $1(1,8 \%)$ Quistes de Chilomastix mesnilli, estos datos se muestran en la Tabla 1 a continuación.

\section{Table 1}

Protozoos identificados.

$\begin{array}{lll}\text { Protozoo identificado } & \text { Número de muestras } \\ \text { Quistes de Endolimax nana } & 25 \\ \text { Quistes de Entamoeba coli } & 19 \\ \text { Quistes de Entamoeba histolytica } & 17 \\ \text { Quistes de Giardia lamblia } & 8 \\ \text { Quistes de Chilomastix mesnilli } & 1 \\ \text { No se observan } & 12\end{array}$

\section{Porcentaje}

$45.5 \%$

$34.5 \%$

$30.9 \%$

$14.5 \%$

$1.8 \%$

$21.8 \%$

Fuente: Propia. 
La prevalencia de protozoos en las muestras fecales analizadas fue de 78,18\% (43/55), de estas muestras 21 que corresponde al 38,2\% estaban monoparasitados, mientras que 22 con el $40 \%$ resultó poliparasitado, y en un $21,8 \%$ de muestras no se observaron protozoos. Dentro de las muestras en las que se identificaron un solo tipo de protozoo, apenas 2 de ellas se presentaron quistes de E. histolytica, que es considerado el protozoo ameboideo patógeno, 14 con presencia única de $E$. nana, y 4 con E. coli, mientras que en 2 muestras se registró la presencia de quistes de G. lamblia únicamente, que es un protozoo flagelado considerado patógeno, como se muestra en la Tabla 2.

Del grupo de muestras en las que se identificaron más de un protozoo, en 22 de estas se encontró la presencia E. histolytica y/o G. lamblia, que son los patógenos de cada grupo de protozoos, teniendo un total de 26 niños y niñas que presenta parasitosis por protozoos patógenos. De las 55 muestras analizadas 29 corresponden a aquellas que presentan protozoos comensales, ver Tabla 2.

\section{Table 2}

Protozoos intestinales identificados.

\begin{tabular}{|c|c|c|c|c|}
\hline \multicolumn{5}{|c|}{ Protozoos intestinales identificados } \\
\hline Resultado & $\begin{array}{l}\text { Número } \\
\text { muestras } \\
\text { reportadas }\end{array}$ & Porcentaje & $\begin{array}{l}\text { Protozoo } \\
\text { identificado }\end{array}$ & $\begin{array}{l}\% \text { de muestras } \\
\text { parasitadas }\end{array}$ \\
\hline \multirow[t]{4}{*}{ Monoparasitado } & 21 & $38,2 \%$ & $\begin{array}{l}\text { Entamoeba } \\
\text { histolytica }\end{array}$ & $3,6 \%$ \\
\hline & & & Giardia lamblia & $3,6 \%$ \\
\hline & & & Endolimax nana* & $25,5 \%$ \\
\hline & & & Entamoeba coli* & $7,3 \%$ \\
\hline \multirow[t]{3}{*}{ Poliparasitado } & 22 & $40 \%$ & $\begin{array}{l}\text { Entamoeba } \\
\text { histolytica y/o }\end{array}$ & $40,0 \%$ \\
\hline & & & Giardia lamblia & $52,7 \%$ \\
\hline & & & $\begin{array}{l}\text { Protozoos } \\
\text { comensales }\end{array}$ & \\
\hline No se observan & 12 & $21,8 \%$ & & \\
\hline TOTAL & 55 & $100 \%$ & & \\
\hline
\end{tabular}

*Protozoos comensales.

Fuente: Propia.

\section{Discusión}

El presente trabajo identificó la presencia de parasitosis por protozoos en un grupo de niños y niñas de 4 a 11 años de edad de la Unidad Educativa Vicente Rocafuerte de la comunidad Cunduana, parroquia Licán del cantón Riobamba, en donde se identificó una frecuencia de parasitosis por protozoos intestinales de $78,18 \%$, cifra de prevalencia más alta si se compara con otros estudios como el de Corrales en 2011, en cual se presenta una prevalencia de $58,5 \%$ en niños de $0-4$ años en Belice [15], de igual manera un 
estudio realizado en Perú por Pajuelo Camacho en 2005, presentó un frecuencia de parasitosis intestinal de 50,9\% [12], sin embargo en estudios realizados en Ecuador en 112 muestras de heces fecales de niños se muestra una prevalencia de protozoos con un $78,3 \%$ [16], que es un dato muy similar al determinado en el presente estudio, que se relaciona con el trabajo de Montero [17] que presenta una frecuencia de 72,9\% de parasitosis por protozoos, este último estudio se realizó en el cantón Calvas de la ciudad de Loja.

Los estudios realizados en varias regiones se pueden relacionar a que la parasitosis intestinal tiene una frecuencia importante en nuestro país.

El protozoo ameboideo patógeno identificado $E$. histolytica muestra una prevalencia de $30,9 \%$, prevalencia un poco más alta si se compara con el estudio de Serpa y otros [18], del año 2014 en cual se indica prevalencia de 24,3\% para este patógeno, el mencionado trabajo se realizó en niños de una escuela de la parroquia Sinincay del cantón Cuenca provincia del Azuay [19], mientras que en indígenas de Bolivia se mostró una prevalencia para E. histolytica de 35,1\%, mostrándose más alta.

En lo referente a G. lambia, flagelado patógeno, se identificó una prevalencia de $14,5 \%$ en el presente trabajo, este dato es menor al mostrado en el trabajo de Luna y otros en 2009 en Bolivia [19] con una prevalencia de 18,7\%, mientras que en el trabajo realizado en Sinincay, Azuay [18] la prevalencia es menor con 6,8\%.

El parásito con mayor prevalencia en el presente trabajo fue $E$. nana encontrándose en el $45,5 \%$ de las muestras parasitadas, este dato es mayor al resultado presentado por Werner y otros en 2014 , con un $38,9 \%$, este protozoo ameboideo es considerado de patogenicidad discutida, sin embargo no se debería restar importancia a su presencia, ya que es trasmitido por las misma vía y condiciones que otros patógenos protozoos relacionados a la presencia de cuadros clínicos, además de que puede ser considerado como un indicador de contaminación fecal de agua y alimentos [13].

No se muestra una diferencia significativa entre los niños monoparasitados $(38,2 \%)$ y los poliparasitados (40\%), a diferencia del estudio de Bracho Mora y otros en Venezuela en el año 2013 [20], en el cual el porcentaje de personas con infecciones mixtas fue de $88,2 \%$, siendo un porcentaje mucho menor los casos con monoparasitosis. Mientras que en Sinsicay Azuay [18], el estudio de muestra un 21,4\% de poliparasitismo con un $35,9 \%$ de monoparasitismo, este último dato se relaciona de mejor manera con lo presentado en la presente investigación.

\section{Conclusiones}

Se determinó una alta prevalencia de parasitosis por protozoos intestinales en los niños y niñas de la Unidad Educativa Vicente Rocafuerte de la comunidad de Cunduana, parroquia Licán, del Cantón Riobamba, provincia de Chimborazo, en junio de 2019, con un valor de $78,2 \%$. El protozoo ameboideo con la mayor prevalencia fue E. nana con un $45,5 \%$, mientras que el protozoo patógeno $E$. histolytica presentó una prevalencia de $30,9 \%$, también se demostró la prevalencia de G. lambia, protozoo flagelado patógeno con el $14,5 \%$. 
El 21,4\% de los niños y niñas parasitadas mostraron una infección mixta, mientras que el $40 \%$ presentó monopasitismo. La falta de una adecuada infraestructura sanitaria, la utilización de incorrectos hábitos de higiene, sumada la contaminación ambiental, son factores que influyen notablemente en la prevalencia de la parasitosis intestinal por protozoos en el grupo estudiado.

\section{Agradecimientos}

A las autoridades de la Unidad Educativa Vicente Rocafuerte de la comunidad de Cunduana, parroquia Licán, del Cantón Riobamba, por las facilidades brindadas para la presente investigación.

\section{Conflicto de Intereses}

No existen conflictos de interés por parte de las autoras.

\section{Declaración de Responsabilidad}

Todos los puntos de vista expresados en el presente escrito son de entera responsabilidad de los autores y no de la Escuela Superior Politécnica del Chimborazo.

\section{Fuentes de Apoyo}

Los autores declaran que las fuentes de apoyo que permitieron la realización de presente trabajo son parte de los recursos asignados al proyecto de investigación del Grupo Generaciones, 'Programa de estudio de patologías nutricionales en niños de primero, segundo, tercero y cuarto año de educación general básica de la ciudad de Riobamba. 2017-2019', de la Facultad de Salud Pública de la ESPOCH.

\section{References}

[1] Chacín-Bonilla L. Las enfermedades parasitarias intestinales como un problema de salud global. Investig Clínica. 2013; 54(1):1-4.

[2] Gamboa MI, Navone GT, Kozubsky L, Costas ME, Cardozo M, Magistrello P. Protozoos intestinales en un asentamiento precario: Manifestaciones clínicas y ambiente. Acta Bioquímica Clínica Latinoam. 2009;43(2):213-8.

[3] Oña-Cisneros F, Costta M, Benavides K, et al. Prevalencia de parásitos intestinales y comparación de métodos diagnósticos en heces en niños escolares de tres parroquias del Distrito Metropolitano de Quito, provincia de Pichincha, Ecuador. Rev Ecu Med Eugenio Espejo. 2015;4(5):16-26.

[4] Rivero de R Z, Calchi L M, Acurero E, et al. Protozoarios y helmintos intestinales en adultos asintomáticos del estado Zulia, Venezuela. Kasmera. 2012; 40(2):186-94.

[5] Rumhein FA, Sánchez J, Requena I, Blanco Y, Devera R. Parasitosis intestinales en escolares relación entre su prevalencia en heces y en el lecho subungueal. Rev Bioméd. 2005;16(4):227-38.

[6] Gómez-Barreno L, Abad-Sojos A, Inga-Salazar G, et al. Presencia de parasitosis intestinal en una comunidad escolar urbano marginal del Ecuador, Ciencia e Investigación Medico Estudiantil Latinoamericana. Cienc E Investig Méd Estud Latinoam. 2017;22(2). 
[7] Cardozo G, Samudio M. Predisposing factors and consequences of intestinal parasitosis in Paraguayan school- aged children. Pediatría Asunción. 2017;;44(2):117-25.

[8] Sánchez PG, de la Fé GR, Rodríguez FHG, Cruz SM, Beltrán GO. Prevalencia de parasitismo intestinal en escolares de 6-11 año. Medisan. 2012;16(4):551-7.

[9] Pino MD, Rinaldo J. Parasitosis intestinal en preescolares y escolares atendidos en el centro médico EsSalud de Celendin, Cajamarca. Horiz Méd Lima. 2016;16(3):35-42.

[10] Laugart EL, García FMR. Aspectos epidemiológicos de las parasitosis intestinales en niños de Vegón de Nutrias, Venezuela. Rev Cuba Hig Epidemiol. 2012; 50(3):330-9.

[11] Fernández NFÁ, Pérez SMH, Valdés LLA, Martín MTA. Hallazgos epidemiológicos en infecciones parasitarias intestinales de un grupo de niños ingresados por diarreas. Rev Cubana Med Trop. 2013;65(1):26-35.

[12] Camacho GP, Roca DL, Perez BP. Estudio de enteroparásitos en el Hospital de Emergencias Pediátricas, Lima-Perú. Rev Medica Hered. 2005;16(3):178-83.

[13] Werner Apt B. Infecciones por parásitos más frecuentes y su manejo. Rev Médica Clínica Las Condes. 2014;25(3):485-528.

[14] Betancourt WQ, Querales LJ. Parásitos Protozoarios entéricos en ambientes acuáticos: Métodos de concentración y detección. Interciencia. 2008;33(6):418-23.

[15] Fuentes CL, García SH, Arencibia RMA, Pérez HA. Parasitismo intestinal infantil: Factores epidemiológicos en Orange Walk, Belice. Rev Cienc Médicas Pinar Río. 2011;15(4):163-78.

[16] Prevalencia de parasitismo intestinal en niños quechuas de zonas rurales montañosas de Ecuador. Rev Panam Salud Pública. 2008;23:125-125.

[17] Bermeo NM. Prevalencia de parasitosis intestinal en niños de la escuela carlos montúfar [Internet] Issuu. [citado 4 de marzo de 2020]. Disponible en: https://issuu.com/jgonzalonso/docs/nuevo_formato_ parasitosis_intestinal

[18] Andrade CAS, Abambari SGV, Rengel MFB. Prevalencia de parasitismo intestinal en los niños de la escuela josé maría astudillo de la parroquia sinincay, 2014. Rev Panor Méd. julio de 2014;8(1):14-9.

[19] Monrroy SL, Jimenez S, Lopez R, Soto M, Benefice E. Prevalencia de parasitismo intestinal en niños y mujeres de comunidades indígenas del río beni. Visión Científica. 2009;1(2):37-46.

[20] Mora AB, de Rodríguez ZR, Cordero ME, et al. Prevalencia de enteroparásitos y anticuerpos IgG antiEntamoeba histolytica en indígenas de la comunidad de Toromo, estado Zulia, Venezuela. Rev Soc Venez Microbiol. 2013;33(2):151-6. 